\title{
On the Application of Sparse Spherical Harmonic Expansion for Fast Antenna Far Field Measurements
}

\author{
Nicolas Mézières, Benjamin Fuchs, Senior Member, IEEE, Laurent Le Coq, Jean-Marie Lerat, Romain Contreres \\ and Gwenn Le Fur
}

\begin{abstract}
The characterization of $3 \mathrm{D}$ antenna radiation patterns is time consuming. The field acquisition duration can be dramatically reduced by leveraging the sparsity of the radiated field spectrum into spherical harmonic basis. Only a small number of measurements points are then necessary to identify the few significant spherical coefficients describing accurately the antenna pattern. In practice, this compressed sensing based procedure requires carefully chosen settings to work efficiently. The fitting to the data and the field sampling strategy are two crucial points in order to ensure a successful fast antenna measurement procedure. Techniques with experimental validations are proposed to ensure a reliable field interpolation. Estimation of savings in acquisition time are also provided to show the interest of the proposed method.
\end{abstract}

Index Terms-Antenna measurements, antenna radiation patterns, compressed sensing, sparse recovery, spherical vector wave expansion

\section{INTRODUCTION}

$\mathbf{T}$ HE increasing complexity of antennas, designed to achieve always more advanced functionalities such as multiple or scanned beams or coded beamforming, leads to challenging measurement tasks. Thus, reducing the field acquisition time has become a research topic of significant practical relevance. The characterization of 3D radiation patterns is nowadays a common measurement procedure. The standard technique [1] requires an important amount of field samples, which is approximately proportional to $\left(k r_{0}\right)^{2}, k$ being the wavenumber and $r_{0}$ the radius of the minimum sphere enclosing the radiating structure. Recently, the sparse expansion of the field radiated by antennas into spherical harmonic basis has been shown to greatly reduce the number of field samples, leading to important decreases in acquisition time, as shown in [2]-[5]. This fast measurement procedure, combined to the associated post-processing method, needs a proper tuning to ensure a reliable field interpolation.

In this letter, considerations for the fast antenna characterization via sparse spherical harmonic expansion are numerically investigated and validated on experimental data. More specifically, the choice of the error tolerance parameter that controls

Manuscript received xx, 2019; revised xx, 2020.

This work was carried out in the frame of a CNES and LNE grant and is supported in part by the European Union through the European Regional Development Fund (ERDF), and by the french region of Brittany, Ministry of Higher Education and Research, Rennes Métropole and Conseil Départemental 35, through the CPER Project SOPHIE / STIC \& Ondes.

N. Mézières is with the IETR, CNES and LNE (e-mail: nicolas.mezieres@univ-rennes1.fr). B. Fuchs and L. Le Coq are with the IETR, UMR CNRS 6164, Rennes, France. J.-M. Lerat is with the LNE, Trappes, France. R. Contreres and G. Le Fur are with the CNES, Toulouse, France.

Digital Object Identifier the fitting to the data, the field sampling point number and their positions are addressed. Estimations of time savings in the field acquisition phase are provided to demonstrate the interest of the approach.

\section{Sparse Spherical HARMONIC Expansion}

\section{A. Spherical Harmonic Expansion}

The electric far-field $\mathbf{E}(\theta, \varphi)$, radiated outside the sphere enclosing all sources can be expanded into Vector Spherical Harmonics (VSH) basis as follows [1]:

$$
\mathbf{E}(\theta, \varphi)=\frac{k}{\sqrt{\eta}} \sum_{s=1}^{2} \sum_{n=1}^{\infty} \sum_{m=-n}^{n} Q_{s m n} \mathbf{F}_{s m n}(\theta, \varphi)
$$

where $\eta$ is the admittance of the propagation medium, $Q_{s m n}$ are the spherical coefficients and $\mathbf{F}_{s m n}$ the VSH. To formalize the problem, the directions $(\theta, \varphi)$ are discretized and the infinite series in (1) is truncated to $N$, where [1] :

$$
N=\left\lfloor k r_{0}\right\rfloor+10 \text {. }
$$

where $k$ is the wavenumber and $r_{0}$ the radius of the minimal sphere enclosing the antenna. After discretization of the directions $(\theta, \varphi),(1)$ can be expressed as a linear system :

$$
\mathbf{y}=\mathbf{A x}
$$

where the vector $\mathbf{x}$ of size $N_{c}$ contains the spherical coefficients $Q_{s m n}$. The vector $\mathbf{y}$ of size $M$ gathers the values of the measured electric far-field $\mathbf{y}_{j}=\mathbf{E}\left(\theta_{j}, \varphi_{j}\right)$. The matrix A corresponds to the harmonics $\mathbf{F}_{s m n}$, each column being associated to a triplet $(s, m, n)$ and each row to a position $\left(\theta_{j}, \varphi_{j}\right)$. The standard method described in [1] requires a (high) number of measurement points $M_{\mathrm{H}}=2(2 N+1)(N+1)$ to analytically retrieve the spherical coefficients. However, antenna radiation patterns can be accurately described from a limited number of spherical harmonics, as shown in [2]-[5]. This sparse vector $\mathbf{x}$ can then be identified from a reduced number of measurements $M$.

\section{B. Sparse Spherical Coefficients Reconstruction}

The determination of the sparse spherical coefficient vector x of size $N_{c}$ from a small number $M\left(<N_{c}\right)$ of field samples $\mathbf{y}_{j}$ can be achieved by solving the following optimization problem, known as Basis Pursuit DeNoising (BPDN) [6]:

$$
\min _{\mathbf{x}}\|\mathbf{x}\|_{1} \text { s.t. }\|\mathbf{A x}-\mathbf{y}\|_{2} \leq \sigma
$$

where the parameter $\sigma>0$ controls the fitting to the data contained in $\mathbf{y}$ and is known as error tolerance. Note that the 
BPDN problem (4) is a specific convex optimization problem, belonging to sparse recovery category, that can be solved efficiently using many readily available routines, such as the SPGL1 algorithm [7], [8]. It takes about 1 minute to solve a problem involving around 30.000 unknowns on a Intel Xeon E5-2609 v2.

Two degrees of freedom strongly impact the resolution of (4): the value of $\sigma$ and the field sampling positions $\mathbf{y}_{j}$. Their choices are crucial to converge to a correct solution and then ensuring a trustworthy field interpolation. These points are addressed in sections III and IV.

\section{Spherical Field Samplings}

We consider 3 sampling strategies, showed on Fig. 1:

- the equiangular sampling, defined by $\delta \theta=\delta \varphi$. This technique produces oversampling near the poles but enables to identify analytically the spherical coefficients using a Fourier Transform [1].

- the igloo sampling, defined by $\delta \varphi=\frac{\delta \theta}{\sin \theta}$ [4]. It allows a faster scan of the sphere, especially for step-by-step measurements, and provides a regular distribution of the points over the sphere.

- the Fibonacci's sampling, or spiral sampling, which is well suited to measurement on-the-fly and provides good results for sparse reconstruction, as showed in [5]. The following formulation will be used; the $j$-th point among $K$ ones is defined by $\varphi_{j}=2 \pi \frac{1+\sqrt{5}}{2}(j-1), \theta_{j}=$ $\arccos \left(1-\frac{2(j-1)}{K}\right)$.

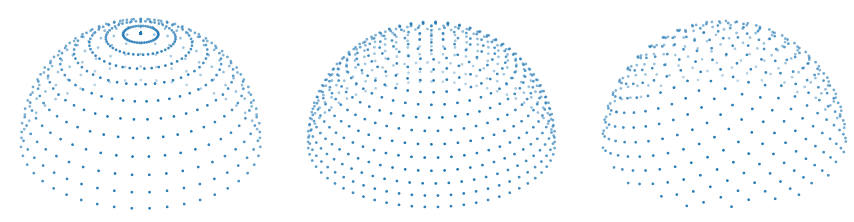

Fig. 1. From left to right: Equiangular sampling, Igloo sampling and spiral/Fibonacci's sampling over the hemisphere.

\section{ON THE Number OF FIELDS SAMPLES}

The determination of the minimum number of field samples required to successfully recover the sparse spherical harmonic spectrum is essential for fast antenna measurements. Theoretical results on sparse recovery performances have been established for Gaussian matrices, and to this end, Phase Transition Diagrams (PTD) have been introduced [9]. Inspired by these works, PTD have been extended for spherical harmonic matrices in [2], [4], [5]. We propose here to go further by drawing random sparse sets of spherical coefficients that better fit the ones of an actual antenna. A concept of effective sparsity will also be introduced, since non-zero but very small magnitude spherical coefficients are negligible.

\section{A. Phase Transition Diagrams}

These diagrams enable to determine the probability of success of the coefficients identification as a function of the sparsity level $\zeta$ of the spherical coefficients and the measurement ratio $\delta$. The sparsity level $\zeta$ is the proportion of non-zero spherical coefficients. The measurement ratio $\delta$ is equal to the number of measurements $M$ over the number of unknowns $N_{c}$. Intuitively, a vector $\mathbf{x}$ with a small $\zeta$ (a small number of non-zero coefficients) can be correctly identified from a small measurement ratio $\delta$.

1) Random Spherical Coefficients: Given a sparsity level $\zeta$ and a measurement ratio $\delta$, we draw numerous random sets of complex spherical coefficients using a normal distribution with zero mean. Standard approaches consider random coefficients drawn with a constant variance. We propose to reduce progressively the variance for high order modes $F_{s m n}$ where $N-10 \leq n \leq N$ in order to be more faithful to real antenna harmonic spectrum.

2) Effective Sparsity: We introduce the effective sparsity as the measure of significant coefficients. Indeed, real-world data are always noisy, there fore low magnitude coefficients are not relevant. The effective sparsity $\zeta_{T}$ with threshold $T$ is

$$
\zeta_{T}=\frac{1}{N_{c}} \#\left\{\frac{\left|\mathbf{x}_{j}\right|}{\|\mathbf{x}\|_{\infty}}>10^{\frac{T}{20}}, j=1, \ldots, N_{c}\right\}
$$

with \# the cardinal operator and $\|\mathbf{x}\|_{\infty}$ the maximum magnitude of x's components.

3) Reconstruction Success: The quantification of the reconstruction performances are done in the radiated field domain instead of the spherical harmonic one as usually done. We compare the interpolated field $\tilde{\mathbf{y}}$ to the reference one $\mathbf{y}$ using the Equivalent Error Signal metric, EES [10], which is defined in $\mathrm{dB}$ as follows

$$
\operatorname{EES}(\mathbf{y}, \tilde{\mathbf{y}})=20 \log _{10}\left(\frac{\|\mathbf{y}-\tilde{\mathbf{y}}\|_{1}}{M\|\mathbf{y}\|_{\infty}}\right) .
$$

\section{B. Numerical Results}

1) Practical Implementation: For each random coefficient set, we compute a reference field $\mathbf{y}$ over a dense equiangular sampling. A subsample is selected for a given measurement ratio $\delta$, and is used to compute an estimation $\widetilde{\mathbf{y}}$ of the field using BPDN. The characterization of the field is considered successful when the EES computed between the reference $y$ and the one computed by BPDN on the subsample $\widetilde{\mathbf{y}}$ is below $-50 \mathrm{~dB}$.

The error tolerance $\sigma$ of the BPDN in (4) is fixed by the following estimation: $\widetilde{\sigma}=\sqrt{M}\|\mathbf{y}\|_{\infty} S$, where $S$ is the maximum expected error in amplitude. We set $S=-60 \mathrm{~dB}$ in this part. Computations are done over the hemisphere and we use the effective sparsity with threshold $T=-40 \mathrm{~dB}, \zeta_{-40}$. Randomly drawing spherical coefficients and thresholding those under $-40 \mathrm{~dB}$ from the highest amplitude mode yields an EES about $-50 \mathrm{~dB}$ between the exact field and the one with thresholded spectrum.

2) Description and Interpretation: The transition curve between successful and failed estimations is contained in a short measurement ratio interval, as shown in Fig. 2. The measurement ratio and effective sparsity level $\zeta_{-40}$ observed for the sparse characterization of the measurements treated in part IV-B of the cavity antenna for Car to $\mathrm{X}$ (C2X) communications, denoted $\mathrm{C} 2 \mathrm{X}$ and the reflectarray, denoted 
RA, are also reported on Fig. 2. As a result, we observe that a measurement ratio of 0.75 should be enough for almost all antennas under our hypothesis. However, positioning errors, alignments errors or various others factors may shift the successful reconstruction zone. The EES metric values obtained

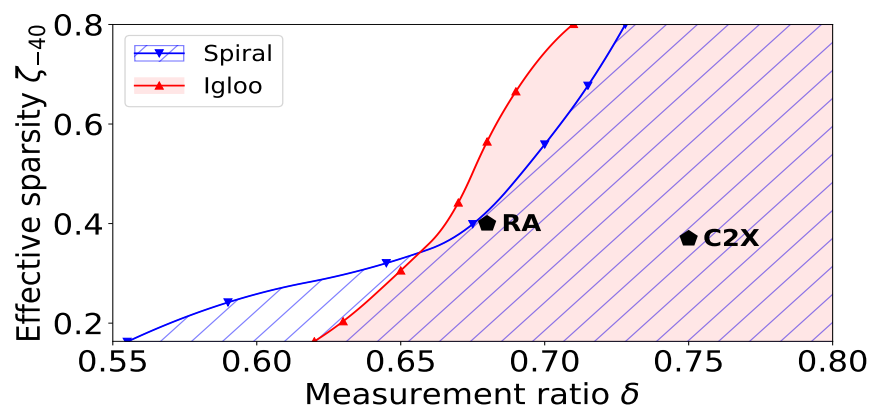

Fig. 2. Successful reconstruction zones, truncation order $N=45\left(N_{c}=\right.$ 4230), Success criterion: EES $<-50 \mathrm{~dB}$, quadratic spline interpolation.

for an effective sparsity level $\zeta_{-40}=0.4$, a level closed to the one observed for both antennas $\mathrm{C} 2 \mathrm{X}$ and $\mathrm{RA}$, are reported on Fig. 3. The dotted lines are the $1 \%$ and $99 \%$ quantiles, meaning that $98 \%$ of the values lie between them. The markers on Fig. 3 and 4 are the averaged values over 200 random spherical coefficient sets. Two main differences can be observed. The transition between failed and successful reconstructions for the igloo sampling is steeper than the one for the spiral sampling. This sudden drop is supported by a broader distribution of the EES values, leading to various situations for each outcome. On the other hand, when the measurement ratio $\delta$ is large enough, the two sampling strategies roughly have the same behavior. For various $N$ and $\zeta_{-40}=0.4$ again, the safe reconstruction zone always occurs around $\delta=0.65$, as illustrated of Fig. 4, indicating that the safe measurement ratio of $\delta=0.75$ can be prolongated to all truncation orders $N$ which can be found in practical situations.

\section{Field Acquisition Time Estimation}

All measurements shown in this paper have been carried out in the IETR centimetric anechoic chamber. The receiver part is composed of a roll-over-azimuth positioning system and the transmitter part has only a roll-axis. The estimation of the field acquisition duration is derived from the values of velocity

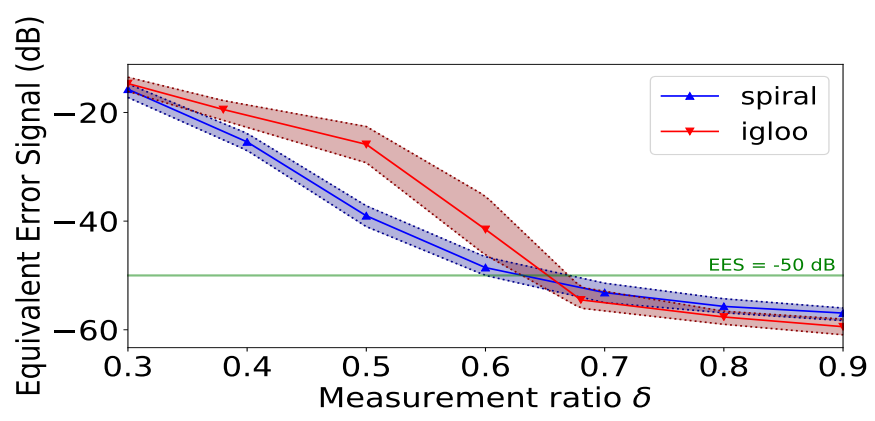

Fig. 3. EES over 200 trials for $N=45$, solid lines $=$ medians, dotted lines $=1 \%$ and $99 \%$ quantiles, $\zeta_{-40}=0.4$, linear interpolation.

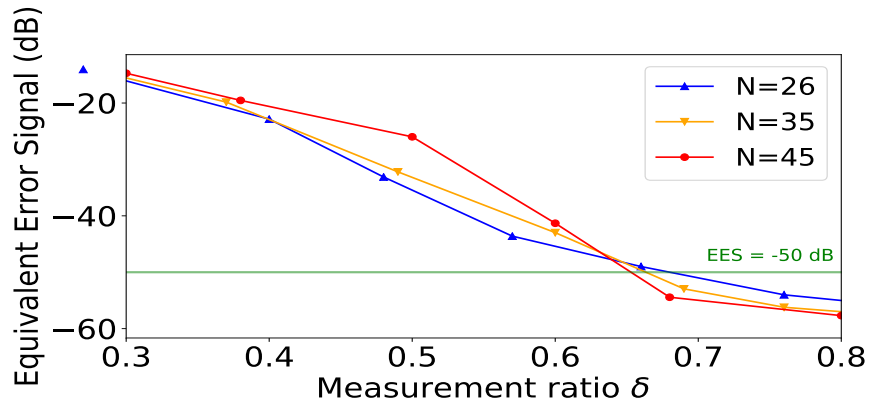

Fig. 4. EES mean value over 200 tries for several truncation orders $N$, Igloo Sampling, $\zeta_{-40}=0.4$, linear interpolation.

TABLE I

ESTIMATED FIELD ACQUISITION TIME FOR IETR CM WAVELENGTH CHAMBER WITH STEP-BY-STEP MEASUREMENTS

\begin{tabular}{|c|c|c|c|c|c|c|}
\hline \multicolumn{4}{|c|}{} & \multicolumn{2}{c|}{ Standard method } & \multicolumn{2}{c|}{ BPDN \& igloo } \\
\hline Ant. & $N$ & $N_{c}$ & Data size $\left(M_{H}\right)$ & Time & Data size & Time \\
\hline C2X & 26 & 1456 & 2862 & $3 \mathrm{~h}$ & 1092 & $1 \mathrm{~h} 25$ \\
\hline RA & 47 & 4606 & 9120 & $7 \mathrm{~h} 45$ & 3112 & $3 \mathrm{~h} 20$ \\
\hline
\end{tabular}

and acceleration of the step-by-step roll and azimuth engines. The transition between a successful and failed reconstruction is very steep, as shown in Fig. 4, meaning that a small amount of time is enough to shift from the bad reconstruction zone to the reliable one. The acquisition time estimations between the standard technique and the sparse characterization with BPDN using an igloo sampling for antennas considered on section IV-B are reported in Table I.

\section{Summary}

The number of data sample $M$ depends on the maximum electrical size of the antenna. A number of $M=0.752 N(N+$ 2 ), $N$ as in (2), where the reduction coefficient 0.75 (also known as them easurement ratio) is achieved by harnessing the sparsity of the spherical coefficients. This number is lower than the one required by the standard approach, $M_{H}=2(2 N+$ $1)(N+1)$. Roughly speaking, this low rate sampling leads to a reduction of about $50 \%$ as compared to the standard approach described in [1] in terms of field acquisition duration.

\section{ERROR TOLERANCE}

The error tolerance parameter $\sigma$ in (4) controls the tradeoff between the sparsity of the spherical coefficients $\|\mathbf{x}\|_{1}$ and their fitting to measured data $\|\mathbf{A x}-\mathbf{y}\|_{2}$. Its choice is discussed in this section.

\section{A. Selection Criterion}

One way to choose $\sigma$ is to apply the L-curve approach proposed for Tikhonov's regularization [11]. This curve helps visualizing the balance between sparsity and data fidelity, defined in our context by $\sigma \mapsto\left(\left\|\mathbf{A} \mathbf{x}_{\sigma}-\mathbf{y}\right\|_{2},\left\|\mathbf{x}_{\sigma}\right\|_{1}\right), \sigma>0$. Using the convex optimization optimal condition on subgradient, we know that $\left\|\mathbf{A x}_{\sigma}-\mathbf{y}\right\|_{2}=\sigma$ whenever $\sigma \leq\|y\|_{2}$. The curve took its name from its $\mathrm{L}$ shape. It has been proved by [11] that the point of maximum curvature at the corner of the $\mathrm{L}$

\section{ACCEPTED MANUSCRIPT}


is a good guess in practical situations. However, this criterion is not perfect, and the maximum curvature point sometimes overshoots the best possible value, as observed in [11].

The computation of the L-curve and its curvature is tedious and time consuming. We can give an estimation of the relevant $\sigma$ interval by using the formula $\widetilde{\sigma}=\sqrt{M}\|\mathbf{y}\|_{\infty} S$, explained in part III-B1. To go further, we adapt an algorithm presented in [12] for Tikhonov's regularization to find the maximum curvature point in an effective way using a golden ratio search by providing the research interval. The curvature can be estimated using Menger's method [12] or parabola method [13]. The error tolerance parameter choice returned by this algorithm is denoted by $\sigma_{\kappa}$.

\section{B. Validations on Experimental Data}

Two different metrics are used to validate the procedure, one based on the $\ell_{1}$ norm, the EES (6), and another one using the $\ell_{2}$ norm, the residual field $\varepsilon$ [14], defined by, using the same notations

$$
\varepsilon(\mathbf{y}, \widetilde{\mathbf{y}})=\frac{\|\mathbf{y}-\tilde{\mathbf{y}}\|_{2}}{\|\mathbf{y}\|_{2}} .
$$

Reference pattern $\mathbf{y}$ is computed on an equiangular sampling with a 2 degrees step from a dense measurement grid. The interpolated field $\widetilde{\mathbf{y}}_{\sigma}$ is derived from solving the BPDN (4) from a coarse igloo. We then plot the L-curve and comparison metric results between $\mathbf{y}$ and $\tilde{\mathbf{y}}$ as functions of $\sigma$ to validate the selection criterion of the error tolerance.

1) Cavity Antenna with radiating slots: We consider a radiating cavity at $6 \mathrm{GHz}$ designed for $\mathrm{C} 2 \mathrm{X}$ communications with radius $r_{0}=13 \mathrm{~cm}$, then a truncation order $N=26$, (or $N_{c}=1456$ coefficients). A sample of size $M=1092$ is used to compute $\widetilde{\mathbf{y}}_{\sigma}$ using BPDN (4), with parameter $\sigma$ for the error tolerance. The formula given in IV-A with $S=-50$ $\mathrm{dB}$ yields $\tilde{\sigma} \approx 1.4 \times 10^{-3}$. The L-curve and its curvature are presented on Fig. 5, the point of maximum curvature can be found around $0.5 \times 10^{-3}$ using Menger approximation method. The greyed out zone corresponds to the aera where the maximum curvature is observed and Fig. 6 shows metrics variations. Both figures also display $\sigma_{\kappa}$, the value returned by the algorithm described in section IV-A.

2) Reflectarray: The procedure is now applied on a reflectarray measured at $12 \mathrm{GHz}$ with a radius $r_{0}=15 \mathrm{~cm}$, then a truncation order $N=47$, (or $N_{c}=4606$ coefficients). The interpolated field $\widetilde{\mathbf{y}}_{\sigma}$ is computed using an igloo sampling with $M=3112$. Estimation $\widetilde{\sigma}$ yields approximately $0.6 \times 10^{-2}$ for $S=-50 \mathrm{~dB}$. Results are displayed on Fig. 7 and 8 .

3) Conclusion: In both cases, the best reconstruction zone is observed around the maximum curvature point. The estimation $\widetilde{\sigma}$ allows to provide a tight search interval for the algorithm to return a relevant choice $\sigma_{\kappa}$ in a convenient time.

\section{CONCLUSION}

The practical aspects of a fast antenna measurement procedure have been addressed in this letter. This procedure takes advantage of the a priori sparsity in the spherical harmonic representation of the radiated field to decrease the number of required measurement points and therefore significantly

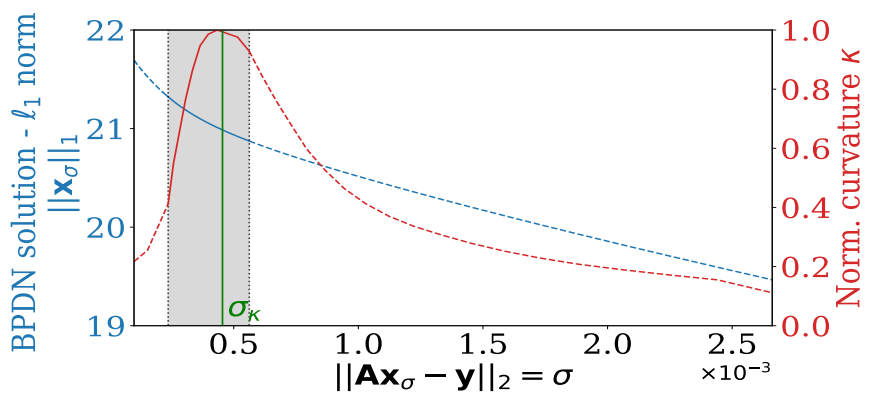

Fig. 5. BPDN L-curve and its curvature (Menger's method) - C2X, $6 \mathrm{GHz}$ Cavity Antenna.

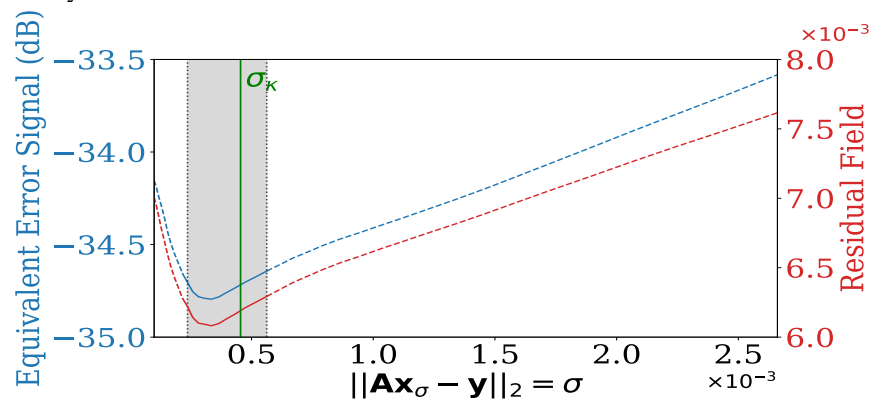

Fig. 6. Metrics values between $\mathbf{E}_{\mathrm{REF}}$ and $\mathbf{E}_{\sigma}-\mathrm{C} 2 \mathrm{X}, 6 \mathrm{GHz}$ Cavity Antenna.

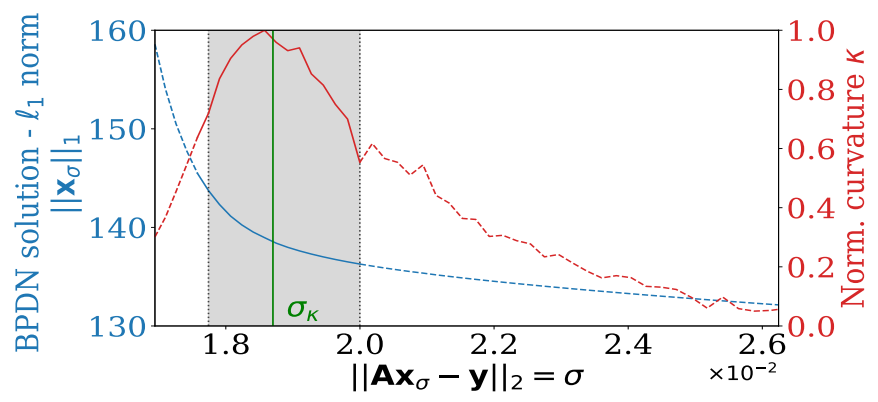

Fig. 7. BPDN L-curve and its curvature (Parabola method) - RA, $12 \mathrm{GHz}$ Reflectarray.

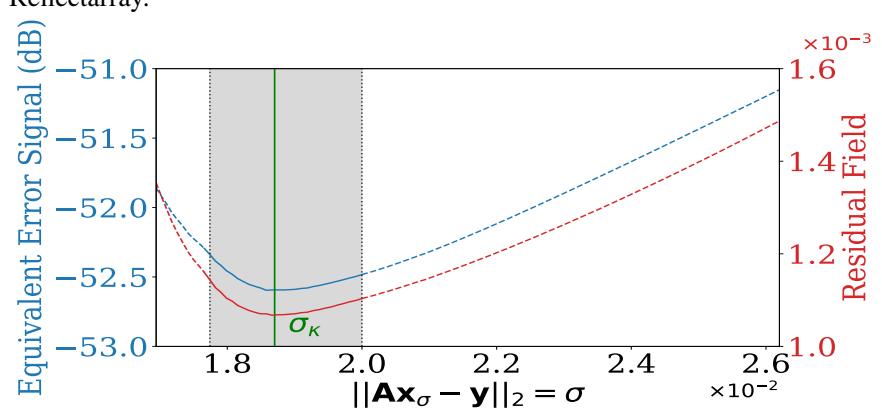

Fig. 8. Metrics values between $\mathbf{E}_{\mathrm{REF}}$ and $\mathbf{E}_{\sigma}-\mathrm{RA}, 12 \mathrm{GHz}$ Reflectarray.

reducing the field acquisition time. The number of necessary field samples for a successful field reconstruction has been thoroughly discussed. An automatic algorithm has been proposed to choose an appropriate parameter to efficiently solve the sparse recovery problem. Experimental validations have been carried out and estimations of the field acquisition time savings have been given, demonstrating the practical interest of the proposed approach. 


\section{REFERENCES}

[1] J. Hald, J. Hansen, F. Jensen, and F. Larsen, Spherical Near Field Antenna Measurements, J. Hansen, Ed. Peter Peregrinus, 1988.

[2] R. Cornelius, D. Heberling, N. Koep, A. Behboodi, and R. Mathar, "Compressed sensing applied to spherical near-field to far-field transformation." Davos: Eur. Conf. Antennas Propag. (EuCAP), 2016.

[3] D. Loschenbrand and C. Mecklenbrauker, "Fast antenna characterization via a sparse spherical multipole expansion." Aachen: 4th International Workshop on Compressed Sensing Theory and its Applications to Radar, Sonar and Remote, 2016.

[4] B. Fuchs, L. Le Coq, S. Rondineau, and M. Migliore, "Fast antenna far field characterization via sparse spherical harmonic expansion," IEEE Trans. Antennas Propag., vol. 65, no. 10, pp. 5503-5510, Oct. 2017.

[5] B. Hofmann, O. Neitz, and T. F. Eibert, "On the minimum number of samples for sparse recovery in spherical antenna near-field measurements," IEEE Trans. on Antennas and Propag., July 2019.

[6] M. Elad, Sparse and Redundant Representations - From Theory to Applications in Signal and Image Processing. Springer, 2010.

[7] E. van den Berg and M. P. Friedlander, "Probing the pareto frontier for basis pursuit solutions," SIAM Journal on Scientific Computing, vol. 31, no. 2, pp. 890-912, 2008. [Online]. Available: https://epubs.siam.org/doi/10.1137/080714488

[8] _ , "SPGL1: A solver for large-scale sparse reconstruction," June 2007.

[9] D. L. Donoho and J. Tanner, "Precise undersampling theorems," Proceedings of the IEEE, vol. 98, no. 6, pp. 913-924, June 2010.

[10] F. Saccardi, F. Rossi, L. Scialacqua, and L. J. Foged, "Truncation error mitigation in free-space automotive partial spherical near field measurements," in 2017 Antenna Measurement Techniques Association Symposium (AMTA), Oct 2017, pp. 1-6.

[11] P. Hansen, "The 1-curve and its use in the numerical treatment of inverse problems," in Computational Inverse Problems in Electrocardiology. WIT Press, 2000.

[12] A. Cultrera and L. Callegaro, "A simple algorithm to find the l-curve corner in the regularization of inverse problems," CoRR, vol. abs/1608.04571, 2016. [Online]. Available: http://arxiv.org/abs/1608.04571

[13] H. Kim and J. Rossignac, "Parabola-based discrete curvature estimation," in 15th Int. Conf. on Computer Graphics, Visualization and Computer Vision (WSCG), Jan. 2006.

[14] G. Giordanengo, M. Righero, F. Vipiana, M. Sabbadini, and G. Vecchi, "Fast antenna testing with reduced near field sampling," IEEE Trans. Antennas Propag., vol. 62, no. 5, pp. 2501-2513, May 2014. 